\title{
Biohidrogenação ruminal e os principais impactos no perfil de ácidos graxos da
}

\section{carne: revisão}

\author{
Ruminal biohidrogenation and main impact on met the fatty acid profile: a review \\ Biohidrogenación ruminal y los principales impactos en el perfil de ácidos grasos de la carne: \\ revisión
}

Recebido: 24/09/2021 | Revisado: 29/09/2021 | Aceito: 01/10/2021 | Publicado: 03/10/2021

\author{
Jocely Gomes de Souza \\ ORCID: https:// orcid.org/0000-0001-6967-161X \\ Universidade Federal da Bahia, Brasil \\ E-mail: jocelygsz@gmail.com \\ Cláudio Vaz Di Mambro Ribeiro \\ ORCID: https://orcid.org/0000-0001-8676-3225 \\ Universidade Federal da Bahia, Brasil \\ E-mail: claudioribeiro@ufba.br
}

\begin{abstract}
Resumo
$\mathrm{Na}$ carne e leite de ruminantes, o perfil dos ácidos graxos (AG) se diferencia pelo maior teor do ácido linoleico conjugado (CLA), molécula com propriedades bioativas. O presente trabalho foi realizado por busca bibliográfica com os objetivos de descrever o processo de BH com suas principias rotas e descrever os principais efeitos da suplementação de lipídios no perfil de AG da carne de ruminantes, ressaltando os potenciais efeitos dos AG de cadeia média (AGCM). O processo de biohidrogenação $(\mathrm{BH})$ ruminal é responsável pela síntese da CLA, e sua manipulação é o foco de muitas pesquisas que tentam aumentar os AG benéficos nos produtos de ruminantes. Com a inibição da BH, aumenta-se o teor de CLA sendo absorvido pelo duodeno. Para tanto, o uso de fontes de AG insaturados e AGCM tem sido estudado. No entanto, existem poucas pesquisas sobre o efeito dos AGCM na BH. Esse AG têm o potencial para aumentar a fluidez das membranas de bactérias gram-positivas, tornando-as ineficientes, diminuindo sua atividade, o que ocasiona uma redução da segunda etapa da BH e, como consequência, um acúmulo do CLA no rúmen. Como fontes principais de AGCM, destacam-se apenas o óleo de coco, dendê e licuri. Mais pesquisas são necessárias para entendermos como elas se comparam a outros métodos de manipulação ruminal e eficiência de melhorar a qualidade dos produtos de ruminantes para o consumo humano, com foco no perfil de AG.
\end{abstract}

Palavras-chave: Microorganismos; Dendê; Licuri; Ácido rumênico.

\begin{abstract}
In the meat and milk of ruminants, the profile of fatty acids (FA) is differentiated by the higher content of conjugated linoleic acid (CLA), a molecule with bioactive properties. The present work was carried out by bibliographical search with the objective of describing the $\mathrm{BH}$ process with its main routes and describing the main effects of lipid supplementation on the FA profile of ruminant meat, highlighting the potential effects of medium chain FA (MCFA). The ruminal biohydrogenation $(\mathrm{BH})$ process is responsible for the synthesis of CLA, and the manipulation of this process is the focus of many research attempting to increase the beneficial FA in ruminant products. By inhibiting ruminal $\mathrm{BH}$, there is an increase the CLA content being absorbed by the duodenum. For this purpose, the use of unsaturated FA and MCFA sources has been studied. However, there is little research on the effect of MCFA in BH. These FA have the potential to increase the fluidity of gram-positive bacterial membranes, making them inefficient, decreasing their activity, which causes a reduction in the second stage of $\mathrm{BH}$ and, as a consequence, an accumulation of CLA in the rumen. As main sources of MCFA, only coconut oil, palm oil and licuri stand out. Further research is needed to understand how they are compared to other methods of ruminal manipulation and efficiency to improve the quality of ruminant products for human consumption, focusing on the FA profile.
\end{abstract}

Keywords: Microorganisms; Palm; Licuri; Rumenic acid.

\section{Resumen}

En la carne y la leche de rumiantes, el perfil de ácidos grasos (AG) difiere por el mayor contenido de ácido linoleico conjugado (CLA), una molécula con propiedades bioactivas. El presente trabajo fue realizado por investigación bibliográfica con el objetivo de describir el proceso BH con sus principales rutas y describir los principales efectos de la suplementación lipídica sobre el perfil AG de la carne de rumiantes, destacando los efectos potenciales de la AG de cadena media (AGCM). El proceso de biohidrogenación ruminal $(\mathrm{BH})$ es responsable de la síntesis de CLA, y su manipulación es el foco de muchos estudios que intentan aumentar la AG benéfica en productos de rumiantes. Con la 
inhibición de BH, el contenido de CLA aumenta siendo absorbido por el duodeno. Para ello, se ha estudiado el uso de fuentes de AG insaturadas y AGCM. Sin embargo, hay poca investigación sobre el efecto de la AGCM en BH. Esta AG tiene el potencial de aumentar la fluidez de las membranas de las bacterias grampositivas, haciéndolas ineficientes, disminuyendo su actividad, lo que provoca una reducción en la segunda etapa de $\mathrm{BH}$, como consecuencia, una acumulación de CLA en el rumen. Como principales fuentes de AGCM, solo destacan el aceite de coco, de palma y licuri. Se necesita más investigación para comprender cómo se comparan con otros métodos de manipulación ruminal y eficiencia para mejorar la calidad de los productos de rumiantes para el consumo humano, centrándose en el perfil de AG.

Palabras clave: Microorganismos; Dendê; Licuri; Ácido ruménico.

\section{Introdução}

Os ácidos graxos (AG) são componentes energéticos na nutrição animal e sua ingestão é na forma esterificada, principalmente de triglicerídeos, a qual também é a forma de reserva em que se encontram os AG no tecido adiposo do animal. Os triglicerídeos são formados por três moléculas de AG condensadas a uma molécula de glicerol. Em ruminantes, os triglicerídeos são hidrolizados no rúmen e os $\mathrm{AG}$ resultantes podem passar pelo processo de biohidrogenação (BH). $\mathrm{Na} \mathrm{BH}$ ocorre a isomerização das duplas ligações e adição de hidrogênio nas ligações duplas, o que caracteriza o rúmen como o maior determinante da composição de AG nos produtos de ruminantes. A regulação da composição lipídica do tecido adiposo e muscular (carne e do leite de ruminantes) pode ser manipulada pelas fontes de AG dietéticos para obter produtos ricos em específicos ácido graxos (Givens et al., 2006), dentre estes o ácido linoleico conjugado (CLA).

O CLA refere-se a um grupo de isômeros posicionais e geométricos do ácido linoleico, porém com ligações duplas conjugadas. Dois isômeros de CLA, o cis-9, trans-11 e o trans-10, cis-12, foram descritos como os isômeros mais ativos biologicamente (Bauman et al., 2001), provenientes da BH parcial de AG no rúmen (Bernard et al., 2009). A alteração nas rotas de BH pode resultar em mudanças nas taxas de BH ou inibição de um passo específico (Griinari \& Bauman, 1999). Várias manipulações dietéticas podem resultar em alteração de rota, tais como: aumento da disponibilidade ruminal de AG insaturados, aumento da fermentação da dieta através de concentrados, fontes de amido rapidamente fermentável, diminuição do fornecimento de fibra efetiva com foragem de partículas pequenas, fornecimento de ionóforos, dentre outros (Van Soest, 1994). Muitas dessas modificações dietéticas podem causar impacto na ruminação, e é bem reconhecida a importância do funcionamento normal do rúmen no desempenho animal.

Os ácidos de cadeia média (AGCM) exercem efeito antimicrobiano (Hristov et al., 2011), provavelmente pela dissociação desses AG na célula bacteriana (Goel et al., 2009). Altos níveis de ácido linoleico inibem a BH do trans-11 18:1 (McKain et al., 2010), sugerindo que as bactérias do segundo passo da BH são mais sensíveis aos AG insaturados. As propriedades físicas dos AG insaturados e de cadeia média são similares. Dessa forma, é possível que possam atuar de maneira semelhante sobre os microrganismos ruminais, aumentando o fluxo de intermediários da BH para o duodeno.

O fornecimento de AGCM poderia reduzir a BH total de fontes de AG insaturados, resultando em um possível aumento de CLA nos produtos de ruminantes. Porém pouco se sabe sobre a interação entre diferentes perfis de AG. Assim, destaca-se a necessidade de estudar o fornecimento de fontes AGCM e seu efeito no metabolismo ruminal, bem como o efeito da alteração das dietas na gordura do leite.

\section{Metodologia}

O presente trabalho foi realizado por busca bibliográfica em revistas científicas as quais possuem revisão por pares. Os objetivos da revisão foram descrever o processo de $\mathrm{BH}$ com suas principias rotas em ruminantes e obter um sumário dos principais efeitos da suplementação de fontes de lipídios no perfil de AG da carne de ruminantes, ressaltando os potenciais efeitos dos AGCM nesse processo. 


\section{Resultados e Discussão}

Os principais temas abordados sobre a BH ruminal foram: a descrição do mecanismo, os microorganismos, seus produtos e a associação com a saúde humana e a suplementação de AG para ruminantes.

\subsection{Mecanismos da biohidrogenação ruminal}

O ambiente ruminal é caracterizado por conter 1010 de bactérias, 107 protozoários, 106 de fungos e leveduras $/ \mathrm{mL}$ de líquido ruminal, temperatura entre 38 a $39^{\circ} \mathrm{C}, \mathrm{pH}$ normal entre 6,0 e 6,7 e potencial redox de -150 a $-350 \mathrm{mV}$. Alterações destas condições podem influenciar a população microbiana e os seus produtos de fermentação (Buccioni et al., 2012). Os AG são compostos reduzidos e contém baixo nível de oxigênio, não fornecendo energia para a fermentação ruminal (Van Soest, 1994). Eles são incorporados na parede celular das bactérias ruminais (Emmanuel, 1974) e a microbiota pode sintetizá-los, incluindo AG de cadeia ímpar e ramificada (Kim et al., 2005).

O teor de lipídios das bactérias (presente principalmente nas membranas) é em torno de $10 \%$ do seu peso seco, sendo constituído de fosfolipídios (30 a 40\%), AG não esterificados (em torno de 40\%) e outras moléculas solúveis em éter, que incluem lipídios neutros (triglicerídeos) e lipídios não saponificáveis (Kozloski, 2011). A degradação de lipídios no ambiente anaeróbico do rúmen é significativamente menor do que em ambientes aeróbios, em decorrência do pequeno potencial de oxidação causada pela baixa concentração de oxigênio (Newbold, 1996). No entanto, um metabolismo importante ocorre quando moléculas de lipídios são alteradas através dos fenômenos de lipólise e BH.

No ambiente ruminal existem dois grandes grupos de bactérias, as gram-positivas e gram-negativas. As bactérias gram-negativas possuem membrana externa, tornando-as mais resistente aos componentes do ambiente ruminal. Determinados AG, especialmente os poliinsaturados, são tóxicos aos microrganismos ruminais. Os microrganismos mais susceptíveis são as bactérias gram-positivas, metanogênicas e protozoários. A toxicidade é relacionada à natureza anfifílica dos AG, isto é, àqueles que são solúveis tanto em solventes orgânicos como em água, são mais tóxicos. Tais ácidos incluem AGCM e AG poliinsaturados de cadeia longa. Assim, a toxicidade parece estar naqueles AG que tem solubilidade maior em água e membranas celulares, com potencial para romper as estruturas das membranas. Os AG se associam com superfícies hidrofóbicas das partículas de alimento, o que explica a baixa toxidade das gorduras quando o animal é alimentado com rações ricas em volumosos (Berchielli et al., 2011).

O processo de BH é responsável pelo aumento da proporção de AG saturados atingindo o duodeno de ruminantes e pela síntese ruminal do CLA e ácido vacênico, os quais são eventualmente incorporados na carne e leite de ruminantes. O processo de BH consiste em dois eventos distintos: lipólise e a biohidrogenação propriamente dita (ou hidrogenação).

\subsubsection{Lipólise}

O primeiro passo do metabolismo de lipídios no rúmen é a hidrólise dos AG ou lipólise (Figura 1), que é realizada majoritariamente pelas bactérias. Os protozoários não estão envolvidos em grande parte na hidrólise, com exceção dos fosfolípidos (Doreau \& Ferlay, 1994). Embora a BH seja alta acima de 90\%, a intensidade desse processo depende das características das fontes de AG, tempo de retenção dessas fontes no rúmen e características da população microbiana (Allen, 2000).

Como o grupo carboxílico livre é necessário para a hidrogenação dos AG insaturados, a lipólise é um pré-requisito para a hidrogenação. Os lipídios esterificados são hidrolisados extensivamente por lipases microbianas após o consumo, causando a liberação da AG constituinte (Jenkins, 1993). 
Figura 1. Esquema de lipólise e biohidrogenação.

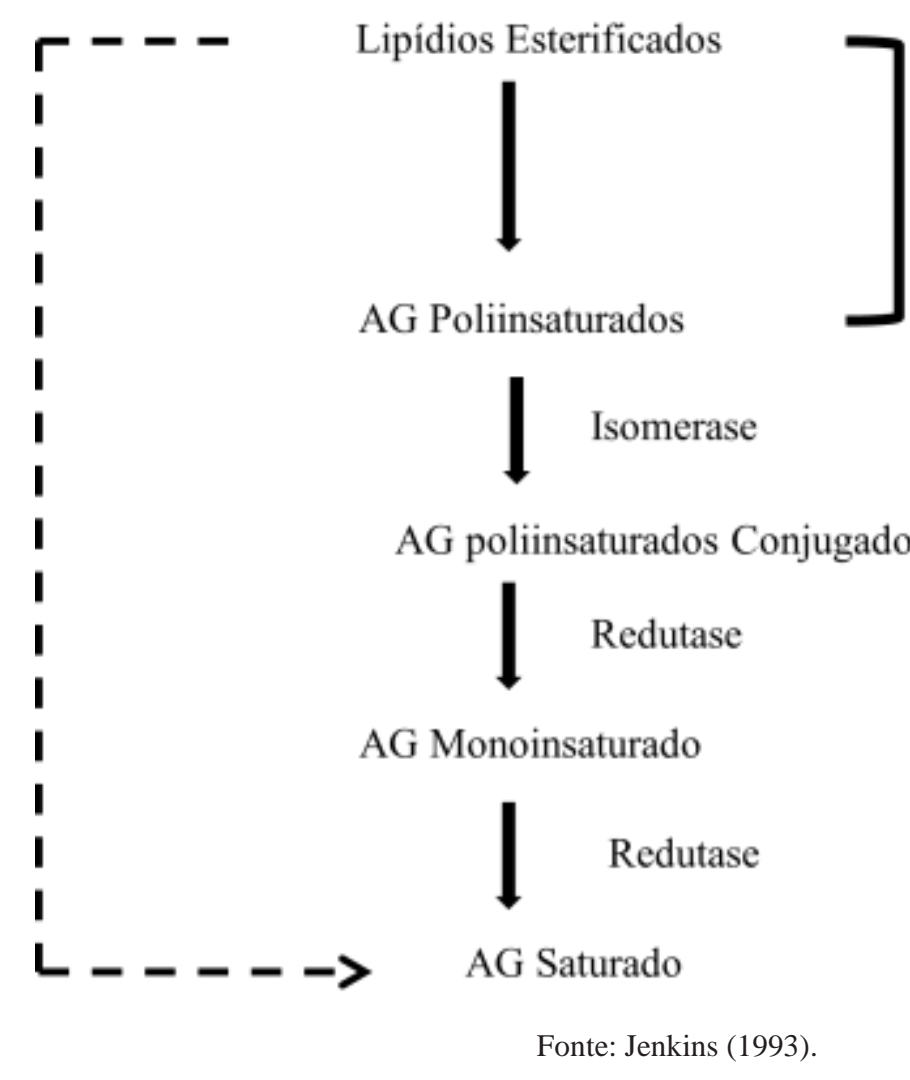

A taxa de lipólise é um fator importante na determinação dos produtos de BH porque altera a liberação e posterior concentração de AG que sofrem de hidrogenação. Como a concentração de alguns AG ode inibir sua hidrogenação (Noble et al., 1974), a taxa de lipólise pode alterar a concentração de seus intermediários. Esses autores também observaram uma hidrogenação mais lenta e menos completa de 18:2 com concentração crescente de trilinoleína. Concluíram que o mecanismo de $\mathrm{BH}$ de 18:2 proveniente do triglicerídeo pode diferir do18:2 ofertado na forma livre, o que demonstra que a forma dos AG suplementados aos ruminantes tem impacto sobre $\mathrm{BH}$.

\subsubsection{Hidrogenação}

A BH depende da concentração de hidrogênio no rúmen, onde somente 1 a $2 \%$ do hidrogênio metabólico é usado para este processo (Czerkawski, 1984). Jenkins et al. (2008) demonstraram que a remoção de hidrogênio através da síntese de metano é cerca de 25 vezes mais eficiente do que via AG. A teoria mais aceita atualmente afirma que a hidrogenação dos AG atua como uma ferramenta de controle da fluidez das membranas celulares dos microrganismos e, consequentemente, da sua permeabilidade (Dehority, 2003). Nessa teoria é sugerido que os AG insaturados inibem o crescimento bacteriano e a BH reverte esse efeito.

A biohidrogenação propriamente dita é um processo intracelular composto por dois sistemas separados, no qual o primeiro passo é a hidrogenação do 18:2 a um ácido octadecenóico (monoeneo), e o segundo passo é a saturação do monoeno ao ácido esteárico (Polan et al., 1964). O primeiro passo da hidrogenação do 18:2 para o ácido esteárico compreende duas reações; a primeira é a produção de um ácido conjugado (cis-9, trans-11 18:2), que é extremamente rápido em comparação com a BH geral (Kepler et al., 1966). A segunda reação produz o ácido vacênico, mais saturado (Noble et al., 1974). O ácido linoleico (cis-9, cis-12), na maior parte, é convertido ao ácido conjugado cis/trans 18:2 dentro de 0,5 horas, e com a continuidade da BH, há um acúmulo do ácido vacênico (trans-18:1) pela hidrogenação do ácido conjugado cis/trans 18:2. 
O segundo passo de BH é a conversão do cis/trans 18:1 para o ácido esteárico, que aumenta rapidamente após altos níveis de cis/trans 18:1 se acumular (Polan et al., 1964) e pode exigir sinergismo de microrganismos ruminais (Polan et al., 1964). Essas reações parecem ter taxas de controle de substratos e também podem ser reguladas por diferentes fatores. Noble et al. (1974) observaram que um acúmulo de grandes quantidades de VA foi precedido em todas as instâncias pelo acúmulo de cis-9, trans-11 18:2, o qual aumentou com a crescente concentração inicial de 18:2 e poderia estar associado à diminuição da taxa de aparecimento de VA. Esses autores sugeriram que fatores diferentes da quantidade de 18:2 podem estar envolvidos na inibição da conversão de 18:1 a 18:0, uma vez que observaram uma inibição persistente após a concentração de 18:2 ter sido reduzida para uma quantidade insignificante. No entanto, essa inibição persistente pode ser atribuída à readaptação dos microrganismos que podem ter sido afetados por altas concentrações de 18:2.

A BH do 18:3 também produz trans-18:1. A primeira reação é uma isomerização, seguida por uma hidrogenação, produzindo cis-9, trans-11, cis-15 18:3 e trans-11, cis-15 18:2, respectivamente. O último AG pode ser BH tanto para trans-11, trans-15, cis-15 18:1 FA. Os trans- e cis-15 18:1 são considerados verdadeiros produtos de BH porque não podem ser mais hidrogenados (Harfoot \& Hazlewood, 1997).

\subsection{Microrganismos}

A compreensão dos microrganismos ruminais envolvidos na $\mathrm{BH}$ de $\mathrm{AG}$ em diferentes alimentos para animais e as vias metabólicas que empregam neste processo são essenciais para qualquer estratégia que visa melhorar os atributos nutricionais de lipídios em produtos de ruminantes (McSweeney \& Mackie, 2012).

Os microrganismos que exercem papel central no metabolismo de AG no rúmen são os protozoários ciliados, que armazenam AG insaturados (Devillard et al., 2006) e várias espécies de bactérias (Butyrivibrio fibrisolvens, Butyrivibrio proteoclasticum, Propionibacterium acnes, Selenomonas ruminantium, Enterococcus faecium, Streptococcus bovis, Staphylococcus sp., e Flavobacterium sp.), prioritariamente envolvidas na BH (McKain et al., 2010).

\subsubsection{Bactérias}

Estudos de Mckain et al. (2010) indicam que há provavelmente três mecanismos para o metabolismo de AG insaturados nas bactérias ruminais que fazem a BH: 1) redução dos isômeros geométricos do cis-9, trans-11 18:2 para trans11-18:1; 2) redução dos isômeros geométricos do trans-10, cis-12 18:2 para uma mistura de isômeros do 18:1 com ligações duplas nos carbonos 10 e 12,3) apenas encontrado em Butyrivibrio proteoclasticus, que reduz uma gama de AG octadecamonoenóicos incluindo trans-10 18: 1, a 18: 0. Os estudos indicaram também que Propionibacterium acnes hidrogena 18:1 para obter substratos de 18:0 como produto final (Figura 2). Estes estudos também fornecem informações sobre os prováveis caminhos no metabolismo de AG (Devillard et al., 2007).

As espécies de bactérias ruminais mais ativas envolvidas na $\mathrm{BH}$ dos $\mathrm{AG}$ de 18 carbonos pertencem ao grupo Butyrivibrio, no qual todas as bactérias formam o CLA a partir de ácido linoleico, enquanto apenas o Clostridium proteoclasticum é capaz de converter o trans-11 18:1 a 18:0 (Polan et al, 1964; Kemp et al., 1975). Recentemente, o $C$. proteoclasticum foi reclassificado como Butyrivibrio proteoclasticus (Moon et al., 2008). 
Figura 2. Papel da Butyrivibrio spp., Propionibacterium acnes e Butyrivibrio proteoclasticum sobre o metabolismo dos ácidos graxos insaturados linoleico e oleico.

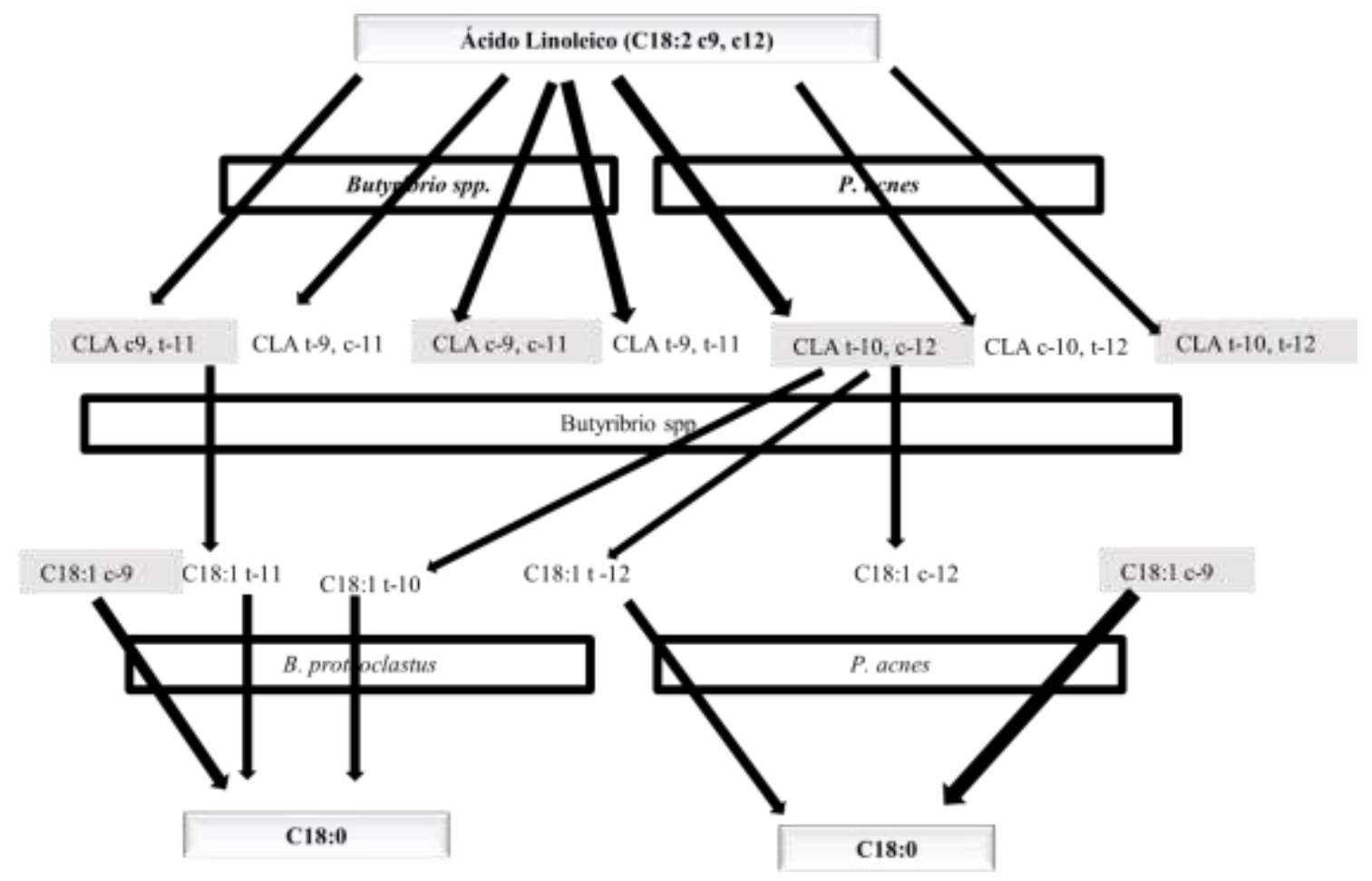

Fonte: McKain et al. (2010) e Wallace et al. (2006), modificado por Buccioni et al. (2012).

As bactérias ruminais envolvidas com a principal rota de BH são dispostas em dois diferentes grupos (A e B) com base em suas ações metabólicas (Kemp \& Lander, 1984). Para que haja BH completa dos AGPI da dieta, torna-se necessária a ação de ambos os grupos. O grupo A, composto principalmente por bactérias fibrolíticas butirogênicas (B. fibrisolvens, Ruminococcus albus, R. flavifaciens etc), é extremamente eficiente em hidrogenar os AG poliinsaturados livres no rúmen, tendo como principal produto intermediário o ácido rumênico (cis-9, trans-11 CLA), e como produto final o ácido vacênico (trans-11 18:1). O grupo B é representado pelo Fusocillus spp. e pelo Clostridium proteoclasticum, reclassificado como Butyrivibrio proteoclasticus. Atualmente, estas são as únicas bactérias isoladas no rúmen capazes de converter AG poliinsaturados a ácido esteárico (Buccioni et al., 2012).

A Anaerovibrio lipolytica é uma bactéria ruminal que produz duas enzimas hidrolíticas. Uma é a esterase ligada às células e a outra é uma lipase extracelular (Harfoot, 1978). A lipase mostra atividade em relação a triglicerídeos e AG esterificados. Hespell e O'Bryan-Shah (1988) examinaram a atividade esterase de várias bactérias ruminais, incluindo 30 cepas de Butyrivibrio fibrisolvens. Todas mostraram possuir alguma atividade esterase, mas algumas espécies podem hidrolisar ésteres com AG de cadeia longa.

\subsubsection{Protozoários}

Mesmo apresentando atividade lipolítica e de hidrogenação, os protozoários são sensíveis aos lipídeos, razão pela qual alguns óleos são utilizados para promover defaunação em ruminantes (Machamüller \& Kreuzer, 1999). O papel dos protozoários na BH não é conclusivo. Contudo, há evidências da participação dos protozoários na BH de AG (Yokoyama \& Johnson, 1993). Sabe-se que a defaunação do rúmen não impede a ocorrência da BH ruminal (Jenkins et al., 2008), sugerindo, então, contribuição limitada dos protozoários nesse processo. 


\subsubsection{Fungos}

Os fungos anaeróbios, por serem numericamente inferiores e metabolicamente menos ativos que as bactérias, apresentam pouca influência sobre o processo de metabolismo de AG. No entanto, eles podem contribuir para o metabolismo lipídico no rúmen devido à capacidade de síntese da enzima $\Delta 9$-desaturase, também conhecida por estearoil-CoA dessaturase, por algumas espécies (por exemplo, Piromyces communis), utilizando ácidos esteárico e vacênico como substratos para a síntese de ácido oleico C18:1 cis- 9 e CLA cis-9 trans-11, respectivamente (Nam \& Garnsworthy, 2007).

\subsection{Produtos da biohidrogenação ruminal e saúde humana}

Há cerca de 40 anos começou a ser dada maior ênfase ao papel da dieta na saúde e em doenças crônicas (Tabela 1), sendo que considerável interesse foi voltado para os lipídios dos alimentos (LIMA et al., 2000). Existem evidências de que O cis-9, trans-11 CLA suprime quimicamente o desenvolvimento de tumor induzido em animais (Parodi, 1999). Alimentos derivados de ruminantes têm uma importante contribuição para o consumo total de gordura (Givens \& Shingfield, 2004) e é a principal fonte de CLA na dieta humana (Lawson et al., 2001).

O CLA foi encontrado em maior concentração em uma ampla variedade de alimentos com carne e leite de ruminantes por Chin et al. (1992). Kepler e Tove (1971) descobriram que o primeiro intermediário na BH do ácido linoleico pelas bactérias ruminais Butyrivibrio fibrisolvens é o 18:2 cis-9, trans-11, o que sugere que os ruminantes podem converter o ácido linoleico em CLA, explicando a maior concentração de CLA em produtos de ruminantes.

O primeiro grupo a relatar o efeito anticarcinogênico do CLA foi o grupo do Dr. Pariza da Universidade de Wisconsin-Madison, nos Estados Unidos (Yavary et al., 2010). O CLA demonstrou potencial para reduzir o câncer em vários órgãos de animais, como a pele, o estômago, o cólon, a glândula mamária e o fígado (Kelley et al., 2007).

Uma explicação para a variedade de atividades biológicas do CLA é que ele é uma mistura de isómeros geométricos e posicionais, com duplas ligações no carbono [9,11], [10,12], [8,10], [7,9] e [11,13]. Embora uma série de isómeros do CLA sejam encontrados em alimentos (Kramer et al., 1998), o principal foco de pesquisa está nos dois principais isômeros, cis-9, trans-11 e trans-10, cis-12. O CLA ocorre naturalmente e consiste principalmente no isómero cis-9, trans-11 (> 80\%) presentes nos alimentos, tais como carne, leite e produtos lácteos (Chin et al., 1992).

Estes dois isômeros podem ter diferentes efeitos sobre o metabolismo e funções celulares, e podem atuar através de diferentes vias de sinalização celular (Wahle et al., 2004). A maioria dos estudos dos efeitos do CLA é sobre a perda ou ganho de gordura, e sobre a composição corporal, pois supostamente melhora a oxidação de gordura e termogênese, e reduz a lipogênese e diferenciação dos pré-adipócitos e proliferação de células cancerígenas (Wang \& Jones, 2004), diminuindo a propensão de doenças cardiovasculares. Além de o CLA ser produzido naturalmente no rúmen por bactérias fermentativas, os ruminantes também sintetizam o CLA a partir do trans-11-18:1 (ácido vacênico) pela enzima delta-9-dessaturase, e este é o AG trans monoinsaturado predominante no tecido adiposo de ruminantes (Parodi, 2003; DHhiman et al., 2005). 
Tabela 1. Histórico dos estudos com ácidos graxos e seus efeitos na saúde humana.

\begin{tabular}{|c|c|c|c|}
\hline Estudo & Período & Resultados & Autores \\
\hline Ausência de Gordura & 1929-19230 & $\begin{array}{c}\text { Diminuição do crescimento, reprodução, doença renal, } \\
\text { fígado gorduroso }\end{array}$ & Bur et al. (1989) \\
\hline Ácidos graxos saturados & 1956 & $\begin{array}{c}\text { Aumento do colesterol sérico, redução do consumo de } \\
\text { gordura. Ácidos graxos insaturados não promoviam } \\
\text { hipercolesterolemia }\end{array}$ & Ahrens et al. (1957) \\
\hline PUFA/AGS & 1956 a 1966 & $\begin{array}{l}\text { Dietas com baixa proporção P:S se associava à alta } \\
\text { incidência de doenças cardiovasculares }\end{array}$ & $\begin{array}{l}\text { Grundy e Bonanome } \\
\text { (1987) }\end{array}$ \\
\hline AGS e Colesterol & 1959 a 1971 & $\begin{array}{c}\text { AGS e colesterol aumentam doenças cardiovasculares } \\
\text { e PUFA exercia diminuição }\end{array}$ & Tuperinen et al. (1979) \\
\hline PUFA/AGS & 1980 & $\begin{array}{c}\text { O risco de morte era inversamente proporcional à } \\
\text { ingestão de PUFAs e diretamente proporcional a } \\
\text { ingestão de colesterol }\end{array}$ & Shekelle et al. (1981) \\
\hline $\begin{array}{l}\mathrm{n}-3 \text { oriundo de óleo de peixe } \\
\text { e dieta normal }\end{array}$ & 1989 & $\begin{array}{l}\text { Redução da mortalidade em } 29 \% \text { consumindo óleo de } \\
\text { peixe (200 a 400g/semana) }\end{array}$ & Burr et al. (1989) \\
\hline $\begin{array}{l}\text { Extratos de carne moída } \\
\text { (CLA) em ratos }\end{array}$ & $1985-1987$ & $\begin{array}{c}\text { Primeiros relatos que o CLA exerce efeitos } \\
\text { anticarcinogênico }\end{array}$ & $\begin{array}{l}\text { Pariza e Hargraves (1985) } \\
\text { Ha et al. (1987) }\end{array}$ \\
\hline $\begin{array}{l}\mathrm{c} 9, \mathrm{t} 11 \text { CLA, } 3 \mathrm{~g} / \mathrm{d} \\
13 \text { semanas em homens com } \\
\text { obesidade abdominal }\end{array}$ & 2004 & $\begin{array}{c}\text { CLA afeta negativamente a resistência à insulina e a } \\
\text { peroxidação lipídica }\end{array}$ & Riserus et al. (2004) \\
\hline $\begin{array}{l}\text { ALA, CLA e VA } \\
\text { Homens e mulheres com } \\
\text { Hipercolesterolemia } \\
(5,68-7,49 \mathrm{mmol} / \mathrm{L})\end{array}$ & 2013 & $\begin{array}{c}\text { A atuação do CLA é sinérgica com outros AG. A } \\
\text { ingestão de um alimento é capaz de afetar o sistema } \\
\text { endocanabinóide }\end{array}$ & Pintus et al. (2013) \\
\hline AGS do leite & 2004 a 2013 & $\begin{array}{l}\text { ÁGS não influenciam no aumento de LDL e estão } \\
\text { inversamente relacionados com doenças cardíacas }\end{array}$ & $\begin{array}{l}\text { Patterson et al. 2013; } \\
\text { Sjogren et al. } 2004\end{array}$ \\
\hline
\end{tabular}

PUFA = ácido graxo poliinsaturado; AGS= ácido graxo saturado; PUFA:AGS = relação PUFA:AGS; CLA= ácido linoleico conjugado; ALA= ácido alfa linolênico; VA= ácido vacênico. Fonte: Autores (2021).

\subsection{Suplementação de ácidos graxos em dietas de ruminantes}

Os lipídeos são utilizados nas dietas para aumentar a energia de rações, resultante de sua elevada densidade calórica para manipular a fermentação ruminal. A fermentação ruminal dos carboidratos estruturais é reduzida pela adição de lipídeos às dietas e o grau de redução depende das fontes de fibra e de lipídeos. A adição de lipídios à dieta pode reduzir as emissões de $\mathrm{CH}_{4}$, o que depende da quantidade suplementada, do grau de insaturação e do comprimento da cadeia do lipídeo. O fornecimento de lipídeos insaturados está associado à redução na produção de $\mathrm{CH}_{4}$ no rúmen por exercer ação deletéria sobre as metanogênicas e protozoários e consumir $\mathrm{H}_{2}$ pelo processo de $\mathrm{BH}$ (Machmüller et al. 1998).

A suplementação através de tortas e farelos vem sendo cada vez mais utilizados, haja visto que estes são coprodutos da indústria de óleo e biodiesel, sendo encontrado em grande escala, grande disponibilidade e geralmente a baixo custo. A taxa de liberação de AG livres é reduzida, pois este se encontra na forma complexada (triglicerídeos e galactolipidios), podendo ocasionar em BH parcial dos AG poliinsaturados, sendo assim, aumentando os intermediários para serem absorvidos no intestino. O fornecimento de torta de dendê e torta de palma decantada resultou em aumento das populações de bactérias celulolíticas (Fibrobacter succinogenes, Ruminococcus flavefeciens, e Ruminococcus albus) e diminuição da densidade de metanogênicas no rúmen de cabras (Abubakr et al., 2014). 


\subsection{1 Ácidos graxos de cadeia média}

Fazem parte dos AGCM o 10:0, 12:0 e 14:0, os quais possuem menor peso molecular em relação aos de cadeia longa, o que resulta em ação mais rápida da lipase pancreática e no intestino delgado, e consequentemente, aumenta a taxa de digestão de AGCM (Bach \& Babayan, 1982). Dentre as principais fontes, destacam-se o óleo de coco (Cocus nucifera) e óleo de dendê (Elaeis guineenses), os quais possuem um perfil de AG semelhante (Tabela 2), incluindo uma maioria de AG saturados (AGS), principalmente o ácido láurico (Dubois et al., 2007).

A oxidação dos AGCM é rápida e pouco influenciada por hormônios e status nutricional do animal (McGarry \& Foster, 1971). Isso ocorre porque AG com comprimento de cadeia de 12 carbonos ou menos entram nas mitocôndrias sem a ajuda de transportadores de membrana. Proporções inferiores a 3,0\% de AGCM (12:0 e 14:0) reduzem em 50\% da emissão de metano in vivo (Machmuller, 2006).

Os ácidos AGCM provenientes da dieta têm potencial para aumentar a fluidez das membranas de bactérias grampositivas, tornando-as ineficientes, diminuindo sua atividade, o que ocasionaria uma redução da segunda etapa da BH como consequência aumentaria o ácido linoleico conjugado. Ainda, a suplementação de AG insaturados de cadeia longa em dietas para vacas diminui os AGCM (10:0, 12:0 e 14:0) e aumentam 18:0 e 18:1 na gordura do leite (Ward et al., 2002). O fornecimento de fontes ricas em AGCM poderia reduzir a BH total de fontes de AG insaturados, resultando em um possível aumento de CLA nos produtos de ruminantes. Porém pouco se sabe sobre a interação entre diferentes perfis de AG, pelas pesquisas serem incipientes.

O objetivo de se concentrar em grandes intermediários e evitar a completa $\mathrm{BH}$ conduziu à persistência de muitas prováveis rotas de BH (Jenkins et al., 2007) e experimentos com diferentes dietas ao longo das décadas (Tabela 3). Ainda existem grandes lacunas sobre o efeito das dietas na composição do perfil de AG em produtos de ruminantes.

A inclusão de AGCM em dietas de ruminantes vêm sendo explorado pelo potencial significativo de reduzir a emissão metano entérico e eficiência da utilização do nitrogênio (Hristov \& Jounay, 2005). Hollmann e Beede (2012) observaram dois efeitos majoritários quando suplementaram vacas de leite com uma de fonte de AGCM (óleo de coco), e em menor efeito com uma fonte de AG de cadeia longa via gordura animal. Primero a introdução abrupta de grande concentração de óleo de coco diminuiu a ingestão de matéria seca e energia e, consequentemente, reduziu os sólidos totais corrigidos para a produção de leite total. Os AGCM, tal como o ácido láurico, demonstraram ter potentes efeitos de defaunação (Faciola \& Broderick, 2013). 
Tabela 2. Perfil de ácidos graxos em óleos vegetais.

\begin{tabular}{lccccc|ccc}
\hline & \multicolumn{3}{c}{ AGCL } & \multicolumn{3}{c}{ AGCM } \\
\cline { 2 - 8 } Ácidos graxos (\%) & OCa & OG & OS & OCz & Ooli & OC & ODe & Opalm \\
\hline C8:0 & - & - & - & - & - & 7,6 & 4,1 & 0,1 \\
C10:0 & - & - & - & 0,01 & - & 5,5 & 3,7 & 0,1 \\
C12:0 & - & 0,02 & - & - & - & 47,7 & 46 & 0,4 \\
C14:0 & 0,1 & 0,09 & 0,1 & - & - & 19,9 & 17,8 & 1,1 \\
C16:0 & 6,7 & 6,2 & 10,8 & 4,6 & 16,5 & - & 8,4 & 43,8 \\
C18:0 & 2,4 & 2,8 & 3,9 & 1,7 & 2,3 & 2,7 & 1,6 & 4,4 \\
C22:0 & - & - & 0,3 & - & 0,15 & - & - & 0,1 \\
C18:1 (n-9) & 11,5 & 28 & 23,9 & 63,4 & 66,4 & 6,2 & 16,4 & 39,1 \\
C18:2 (n-6) & 79 & 62,2 & 52,1 & 19,6 & 16,4 & 1,6 & 3,1 & 10,2 \\
C18:3 (n-3) & 0,15 & 0,16 & 7,8 & 1,2 & 1,6 & - & - & 0,3 \\
C18:3 (n-6) & - & - & - & - & - & - & - & - \\
AGS & 9,3 & 9,4 & 15,7 & 6,3 & 19,4 & 92,1 & 81,9 & 50,4 \\
AGMI & 11,6 & 28,3 & 24,2 & 72,8 & 68,2 & 6,2 & 16,4 & 39,4 \\
AGPI & 79,1 & 62,4 & 59,8 & 20,9 & 18 & 1,6 & 3,1 & 10,5 \\
\hline
\end{tabular}

AGCL= ácidos graxos de cadeia longa; $\mathrm{AGCM}=$ ácidos graxos de cadeia média; $\mathrm{AGS}=$ ácidos graxos saturados AGMI = ácidos graxos monoinsaturados; AGPI = ácidos graxos poliinsaturados; Oca= óleo de cártamo; OG= óleo de girassol; $\mathrm{OCz}=$ óleo de colza; Ooli= óleo de oliva; $\mathrm{OC}=$ óleos de coco; $\mathrm{ODe}=$ óleo de dendê; Opalm= óleo de palma.

Fonte: Dubois et al. (2007) e Orsavova et al. (2015).

Os AGCM exercem efeito antimicrobiano (Hristov et al., 2011), provavelmente pela dissociação desses AG na célula bacteriana (Goel et al., 2009). Altos níveis de ácido linoleico inibem a BH do trans-11 18:1 (McKain et al., 2010), sugerindo que as bactérias do segundo passo da BH são mais sensíveis aos AG insaturados. As propriedades físicas dos AG insaturados e de cadeia média são similares. Dessa forma, é possível que possam atuar de maneira semelhante sobre os microrganismos ruminais, aumentado o fluxo de intermediários da $\mathrm{BH}$ para o duodeno. 
Tabela 3. Efeito dos ácidos graxos da dieta sobre o perfil na carne de ruminantes.

\begin{tabular}{|c|c|c|}
\hline Fonte de lipídio & Resultados & Autor \\
\hline Óleo de cártamo & Aumento do CLA em vários tecidos de Cordeiros & $\begin{array}{l}\text { Mir et al. (2000), Boles et } \\
\text { al. (2005) }\end{array}$ \\
\hline Óleo de soja & Mais efetivo no aumento do CLA no músculo de cordeiros & $\begin{array}{l}\text { Bessa et al. (2005), Santos- } \\
\text { Silva et al. (2004) }\end{array}$ \\
\hline $\begin{array}{l}\text { Óleo de girassol e óleo de } \\
\text { linhaça }\end{array}$ & $\begin{array}{l}\text { Ambos aumentaram o CLA na gordura do músculo, mas maior aumento } \\
\text { com óleo de girassol }\end{array}$ & Bessa et al. (2007) \\
\hline $\begin{array}{l}\text { Óleo de girassol e óleo de } \\
\text { linhaça }\end{array}$ & $\begin{array}{l}\text { Substituição de óleo de girassol por óleo de linhaça reduziu CLA, mas } \\
\text { aumento níveis de n-3 no tecido adiposo de cordeiros }\end{array}$ & Jerónimo et al. (2009) \\
\hline Óleo de peixe & $\begin{array}{l}\text { Aumento dos níveis de ácidos graxos PUFA de cadeia longa (EPA e } \\
\text { DHA) no músculo de cordeiros }\end{array}$ & $\begin{array}{l}\text { Cooper et al. (2004), } \\
\text { Demirel et al. (2004) }\end{array}$ \\
\hline Óleo de palma & Aumento de ácidos graxos indesejáveis no músculo de cordeiros & Castro et al. (2005) \\
\hline Óleo de coco & Redução de AGCC, 16:0 e 18:0 no leite de vacas & Hristov et al. (2009) \\
\hline Óleo de canola & $\begin{array}{l}\text { Aumentou o teor de ácidos graxos n-3 muscular, mas reduziu a oxidação } \\
\text { lipídica no sangue e no músculo LL de cabritos. }\end{array}$ & Karami et al. (2013) \\
\hline Óleo de Linhaça & Aumento de 18:3, n-3 e CLA na carne de bovinos & Nassu et al. (2011) \\
\hline $\begin{array}{l}\text { Óleo de linhaça (OL), } \\
\text { girassol (OG) e soja (OS) }\end{array}$ & $\begin{array}{l}\text { Aumento de n-3 (PUFA) no Longissimus dorsi com óleo de linhaça, } \\
\text { aumento de CLA com OL e OS na carne de bovinos }\end{array}$ & González et al. (2014) \\
\hline Óleo rico em n-3 ou n-6 & $\begin{array}{l}\text { Aumento de ácidos graxos biologicamente ativos, diminuição da relação } \\
\text { n-6:n-3 na carne de bovinos }\end{array}$ & He et al. (2011) \\
\hline Óleo Vegetal e óleo de peixe & Aumento de n-3 e CLA na carne de bovinos & Ducket e Gillis (2010) \\
\hline
\end{tabular}

CLA = ácido linoleico conjugado; EPA= ácido eicosapentanóico; DHA = ácido docosapentanóico; OL= óleo de linhaça; OG= óleo de girassol; OS= óleo de soja.

Fonte: Adaptado de Ribeiro et al. (2011).

\section{Considerações Finais}

A qualidade da carne de ruminantes, em relação ao seu perfil de ácidos graxos, é variável e dependente principalmente da fonte de lipídios suplementada na dieta. Quanto maior o teor de ácido linoleico na dieta, maior o potencial de enriquecimento da carne com CLA. Ainda, fonte de lipídios dietéticos que diminuam a última etapa da biohidrogenação podem aumentar ainda mais o teor de CLA na carne de ruminantes. Dentre estas fontes, os ácidos graxos de cadeia média se destacam por serem fontes encontradas principalmente em países tropicas, incluindo o Brasil. Dentre as fontes mais estudadas, estão o óleo de coco e as tortas de licuri e dendê. A interação entre fontes de ácidos graxos de cadeia média com fontes ácidos de cadeia longa deveria ser o foco de futuras pesquisas visando aumentar o teor de CLA na carne e leite de ruminantes.

\section{Referências}

Abubakr, A., Alimon, A. R., Yaakub, H., Abdullah, N., \& Ivan, M. (2014). Effect of feeding palm oil by-products based diets on total bacteria, cellulolytic bacteria and methanogenic archaea in the rumen of goats. PLoS One, 9(4), 1-6.

Allen, M. S. (200). Effects of diet on short-term regulation of feed intake by lactating dairy cattle. Journal of Dairy Science, 83(7), $1598-1624$.

Bach, A. C., \& Babayan, V. K. (1982). Medium-chain triglycerides: an update. The American Journal of Clinical Nutrition, 36(5), 950-62.

Bauman, D. E., Baumgard, L. H., Corl, B. A., \& Griinari, J. M. (2001). Conjugated linoleic acid (CLA) and the dairy cow. In: Recent Advances in Animal Nutrition - 2001. P. C. Garnsworthy and J. Wiseman, eds. Nottingham University Press, Nottingham, UK.

Berchielli, T. T., Pires, A. V., \& Oliveira, S. G. (2011). Nutrição de Ruminantes. Editora Jaboticabal, Funep.

Bernard, L., Leroux, C., Faulconnier, Y., Durand, D., Shingfield, K. J., \& Chilliard, Y. (2009). Effect of sunflower-seed oil or linseed oil on milk fatty acid secretion and lipogenic gene expression in goats fed hay-based diets. The Journal of dairy research, 76(2), 241-148.

Buccioni, A., Decandia, M., Minieri, S., Molle, G., \& Cabiddu, A. (2012). Lipid metabolism in the rumen: New insights on lipolysis and biohydrogenation with an emphasis on the role of endogenous plant factors. Animal Feed Science and Technology, 174(1), 1-25.

Chin, S. F., Liu, W., Storkson, J. M., Ha, Y. L., \& Pariza, M. W. (1992). Dietary sources of conjugated dienoic isomers of linoleic acid. Journal of Food Composition and Analysis, 5(3), 185-197.

Czerkawski, J.W. Microbial fermentation in the rumen. (1984). Proceedings of the Nutrition Society, 43(2), 101-118. 
Dehorty, B. A. (2003). Rumen Microbiology. Nottingham: Nottingham University Press.

Devillard, E., McIntosh, F. M., Duncan, S. H., \& Wallace, R. J. (2007). Metabolism of linoleic acid by human gut bacteria: different routes for biosynthesis of conjugated linoleic acid. Journal of Bacteriology, 189(6), 2566-2570.

Devillard, E., McIntosh, F. M., Newbold, C. J., \& Wallace, R. J. (2006). Rumen ciliate protozoa contain high concentrations of conjugated linoleic acids and vaccenic acid, yet do not hydrogenate linoleic acid or desaturate stearic acid. British Journal Nutrition, 96(4), 697-704.

Dhiman, T. R., Nam, S. H., \& Ure, A. L. (2005). Factors affecting conjugated linoleic acid content in milk and meat. Critical Reviews in Food Science and Nutrition, 45(6), 463-482.

Doreau, M., \& Ferlay, A. (1994). Digestion and utilisation of fatty acids by ruminants. Animal Feed Science and Technology, 45(3), 379-396.

Emmanuel, B. (1974). On the origin of rumen protozoan fatty acids. Biochimica et Biophysica Acta (BBA), $337(3), 404-413$.

Estrela, C. (2018). Metodologia Científica: Ciência, Ensino, Pesquisa. Editora Artes Médicas.

Faciola, A. P., \& Broderick, G. A. (2013). Effects of feeding lauric acid on ruminal protozoa numbers, fermentation, digestion, and on milk production in dairy cows. Journal Animal Science, 91(5), 2243-2253.

Givens, D. I., Kliem, K. E., \& Gibbs, R. A. (2006). The role of meat as a source of n-3 polyunsaturated fatty acids in the human diet. Meat Science, 74(1), 209-218.

Givens, D. I., \& Shingfield, K. J. (2004). Food derived from animals: the impact of animal nutrition on their nutritive value and ability to sustain long-term health. Nutrition Bull, 29, 325-332.

Goel, G., Arvidsson, K., Vlaeminck, B., Bruggeman, G., Deschepper, K., \& Fievez, V. (2009). Effects of capric acid on rumen methanogenesis and biohydrogenation of linoleic and $\alpha$-linolenic acid. Animal, 3(6), 810-816.

Griinari, J. M., \& Bauman, D. E. (1999). Biosynthesis of conjugated linoleic acid and its incorporation into meat and milk in ruminants. In: Yurawecz, M. P.; Mossoba, M. M.; Kramer, J. K. G.; Pariza, M. W.; Nelson, G. J. Nelson (Ed.) Advances in Conjugated Linoleic Acid Research, Vol. 1. AOCS Press, Champaign, IL.

Harfoot, C. G. (1978). Lipid metabolism in the rumen. Progress in lipid research, 17(1), $21-54$.

Hespell, R.B., \& O’Bryan-Shah, P. J. (1988). Esterase activities in Butyrivibrio fibrisolvens strains. Applied and Environmental Microbiology, 54(8), 19171922 .

Hollman, M., \& Beede, D. K. (2012). Comparison of effects of dietary coconut oil and animal fat blend on lactational performance of Holstein cows fed a high-starch diet. Journal of Dairy Science, 95(3), 1484-1499.

Hristov, A.N., \& Jouany, J.P. (2005). Factors affecting the efficiency of nitrogen utilization in the rumen. In: Nitrogen and phosphorus nutrition of cattle and environment. $\mathrm{CAB}$ International, Wallingford, UK.

Hristov, A. N., Lee, C., Cassidy, T., Long, M., Heyler, K., Corl, B., \& Forster, R. (2011). Effects of lauric and myristic acids on ruminal fermentation, production, and milk fatty acid composition in lactating dairy cows. Journal of Dairy Science, 94(1), 382-395.

Jenkins, T. C., \& Bridges, W.C. (2007). Protection of fatty acids against ruminal biohydrogenation in cattle. European Journal of Lipid Science and Technology, 109(8), 778-789.

Jenkins, T.C., Wallace, R. J., Moate, P. J., \& Mosley, E. E. (2008). Board-invited review: Recent advances in biohydrogenation of unsaturated fatty acids within the rumen microbial ecosystem. Journal of Animal Science, 86(2), 397-412.

Kelley, N.S., Hubbard, N.E., \& Erickson, K.L. (2007). Conjugated linoleic acid isomers and cancer. The Journal of Nutrition, 137(12), 2599-2607.

Kemp, P., Lander, D. J., \& Orpin, C. G. (1984). The lipids of the rumen fungus Piromonas communis. Microbiology, 130(1), $27-37$.

Kemp, P., White, R.W., \& Lander, D. J. (1975). The hydrogenation of unsaturated fatty acids by five bacterial isolates from the sheep rumen, including a new species. Microbiology, 90(1), 100-114.

Kepler, C., \& Tove, S. (1971). Biohydrogenation of unsaturated fatty acids. The Journal Biological Chemistry, 246(16), 5025-5030.

Kepler, C. R., Hirons, K. P., McNeill, J. J., \& Tove, S. B. (1966). Intermediates and products of the biohydrogenation of linoleic acid by Butyrinvibrio fibrisolvens. The Journal Biological Chemistry, 241, 1350-1354.

Kim, E. J., Sandersson, R., Dhanoa, M. S., \& Dewhurst, R. J. (2005). Fatty acid profiles associated with microbial colonization of freshly ingested grass and rumen biohydrogenation. Journal of Dairy Science, 88(9), 3220-3230.

Kozloski, G. V. Bioquímica dos ruminantes. (2011). UFSM.

Kramer, J. K. Distributions of conjugated linoleic acid (CLA) isomers in tissue lipid classes of pigs fed a commercial CLA mixture determined by gas chromatography and silver ion-high-performance liquid chromatography. (1998). Lipids, 33(6), 549-558.

Lawson, R. E., Moss, A. R., \& Givens, D. I. (2001). The role of dairy products in supplying conjugated linoleic acid to man's diet: a review. Nutrition Research Reviews, 14(1), 153-172. 
Lima, F. E. L., Menezes, T. N., Tavares, M. P., Szarfarc, S. C., \& Fisberg, R. M. (2000). Ácidos graxos e doenças cardiovasculasculares: uma revisão. Revista de Nutrição, 13(2), 73-80.

Machamuller, A., \& Kreuzer, M. (1999). Methane suppresion by coconut oil and associated effects on nutrient and energy balance in sheep. Canadian Journal of Animal Science, 79(1), 65-72.

Machmuller, A. (2006). Medium-chain fatty acids and their potential to reduce methanogenesis in domestic ruminants. Agriculture, Ecosystems and Environment., 112(2), 107-114.

Machmuller, A., Ossowski, D. A., Wanner, M., \& Kreuzer, M. (1998). Potential of various fatty feeds to reduce methane release from rumen fermentation in vitro (RUSITEC). Animal Feed Science and Technology, 71(1), 117-130.

McGarry, J. D., \& Foster, D.W. (1971). The regulation of ketogenesis from octanoic acid. The role of the tricarboxylic acid cycle and fatty acid synthesis. The Journal of Biological Chemistry, 246(4), 1149-1159.

McKain, N., Shingfield, K. J., \& Wallace, R. J. (2010). Metabolism of conjugated linoleic acids and 18: 1 fatty acids by ruminal bacteria: products and mechanisms. Microbiology, 156(2), 579-588.

McSweeney, C., \& Mackie, R. (2012). Micro-organisms and ruminant digestion: State of knowdge, trends and future prospects. Background Study Paper, 61. Commission on Genetic Resources for Food and Agriculture.

Moon, C. D., Pacheco, D. M., Kelly, W. J., Leahy, S. C., Li, D., Kopecny, J., \& Attood, G. T. (2008). Reclassification of Clostridium proteoclasticum as Butyrivibrio proteoclasticus comb. nov., a butyrate-producing ruminal bacterium. International Journal Systematic and Evolutionary Microbiology, 58(9), 2041-2045.

Nam, I.S., \& Garnsworthy, P. C. (2007). Biohydrogenation of linoleic acid by rumen fungi compared with rumen bacteria. Journal of Applied microbiology, 103(3), 551-556.

Newbold, C.J. (1996). Probiotics for ruminants. Annales de Zootechnie, 45, 329-335.

Noble, R. C., Moore, J. H., \& Harfoot, C. G. (1974). Observations on the pattern on biohydrogenation of esterified and unesterified linoleic acid in the rumen. Br. J. Nutr. 31, 99-108.

Parodi, P. W. (1999). Conjugated linoleic acid and other anticarcinogenic agents of bovine milk fat. Journal of Dairy Science, 82, 6, $1339-1349$.

Parodi, P. W. (2003). Conjugated linoleic acid in food. In: Sebedio, J. L.; Christie, W.W.; Adlof, R. (Eds). Advances in Conjugated Linoleic Acid Research, Vol. 2, Champaign, IL: AOCS Press.

Polan, C. E., McNeill, J. J., \& Tove, S. B. (1964). Biohydrogenation of unsaturated fatty acids by rumen bacteria. Journal of Bacteriology, 88(4), $1056-1064$. Van Soest, P. J. (1994). Nutritional ecology of the ruminant. Cornell University Press.

Wahle, K. W. J., Heys, S. D., \& Rotondo, D. (2004). Conjugated linoleic acids: Are they beneficial or detrimental to health? Progress in Lipid Research, 43(6), 553-587.

Wang, Y.W., \& Jones, P. J. (2004). Conjugated linoleic acid and obesity control: efficacy and mechanisms. International Journal of Obesity and Related Metabolic Disorders, 28(8), 941-955.

Ward, A.T., Wittenberg, K. M., \& Przybylski, R. (2002). Bovine milk fatty acid profiles produced by feeding diets containing solin, flax and canola. Journal of Dairy Science, 85(5), 1191-1196.

Yavary, A., Hamedi, M., Heshmati, A., \& Haghbin, S. (2010). Retraction: Are conjugated linoleic acid (CLA) isomers good or bad trans fats? Lipid Technology, 22(10), 227-229.

Yokoyama, M. T., \& Johnson, K. A. (1993). Microbiology of the Rumen and Intestine in the Ruminant Animal: Digestive Physiology and Nutrition. D.C. Church, Edited by Prentice Hall, Englewood Cliffs, N.J. 